\title{
Model-Based Transfer Functions for Efficient Visualization of Medical Image Volumes
}

\author{
Daniel Forsberg ${ }^{1,2,3}$, Claes Lundström ${ }^{2,3}$, \\ Mats Andersson ${ }^{1,2}$, and Hans Knutsson ${ }^{1,2}$ \\ 1 Department of Biomedical Engineering, Linköping University, Sweden \\ 2 Center for Medical Image Science and Visualization (CMIV), \\ Linköping University, Sweden \\ 3 Sectra Imtec, Linköping, Sweden
}

\begin{abstract}
The visualization of images with a large dynamic range is a difficult task and this is especially the case for gray-level images. In radiology departments, this will force radiologists to review medical images several times, since the images need to be visualized with several different contrast windows (transfer functions) in order for the full information content of each image to be seen. Previously suggested methods for handling this situation include various approaches using histogram equalization and other methods for processing the image data. However, none of these utilize the underlying human anatomy in the images to control the visualization and the fact that different transfer functions are often only relevant for disjoint anatomical regions.

In this paper, we propose a method for using model-based local transfer functions. It allows the reviewing radiologist to apply multiple transfer functions simultaneously to a medical image volume. This provides the radiologist with a tool for making the review process more efficient, by allowing him/her to review more of the information in a medical image volume with a single visualization. The transfer functions are automatically assigned to different anatomically relevant regions, based upon a model registered to the volume to be visualized. The transfer functions can be either pre-defined or interactively changed by the radiologist during the review process. All of this is achieved without adding any unfamiliar aspects to the radiologist's normal work-flow, when reviewing medical image volumes.
\end{abstract}

\section{Introduction}

The visualization of images with a large dynamic range, i.e. images that have a large value range, is a difficult task and is common in many imaging applications. This also applies to gray-level images, since the human eye has a limited ability to discern different gray-levels and since many display devices are incapable of properly displaying a large number of gray-levels, i.e. there is a limited display range.

A radiology department is a high-pace work environment, where radiologists are under constant pressure to review a never-ending stream of images, and in

This work was funded by the Swedish Research Council, grant 2007-4786.

A. Heyden and F. Kahl (Eds.): SCIA 2011, LNCS 6688, pp. 592 603, 2011.

(C) Springer-Verlag Berlin Heidelberg 2011 
which it is especially important to handle the problem of visualizing gray-level images with a large dynamic range. Despite the digitalization that the whole work-flow has undergone in radiology departments, the review process of the images still remains the same to a large extent, i.e. images are reviewed as graylevel images on an image by image basis. This means that the review process for a whole body CT scan, which can easily generate thousands of images, will require a considerable amount of time.

The issue of a large dynamic range is usually handled by providing the radiologist with functionality that allows him/her to interactively change, what is know as, the contrast window (or the window/level) of the displayed images; hereafter referred to as applying a transfer function. A contrast window is defined as a linear mapping of the pixel values in a certain value range to a given display range. The value range is either determined by a min and a max value or by a width and a center value. This allows him/her to adjust the contrast and the brightness of the visualized images in accordance with either some predefined settings or based upon experience. However, when visualizing images, the radiologist often employs several transfer functions. In the case of a $\mathrm{CT}$ chest scan, this can mean that he/she first scrolls through the image stack with a soft tissue contrast window applied, then with a bone window, and finally with a lung window, see Fig. 11. Thus, the radiologist has to scroll through the stack several times, despite the fact that the different windows are often only relevant to disjoint regions of the body.

The problem of having too large a dynamic range to display is a well-known problem within the medical imaging community [11, and for which a number of different solutions have been proposed. Many of these suggestions are based on histogram equalization and include adaptive histogram equalization (AHE) [12], contrast limiting adaptive histogram equalization (CLAHE) [13] and multi-scale adaptive histogram equalization (MAHE) 6]. Although they enable the radiologist to see more, they are limited in the sense that they rely on a pre-processing step. Unsatisfactory pre-processing might force the radiologist to manually find an optimal set of parameters for each medical image volume in order to produce satisfactory images. Since parameters such as tile size, number of bins and clip limit are unfamiliar to the radiologist and also difficult to master, in practice the only tool for changing the visualization of an image still is a global min-max windowing. See Fig. 2 for an example using CLAHE with different parameter sets.
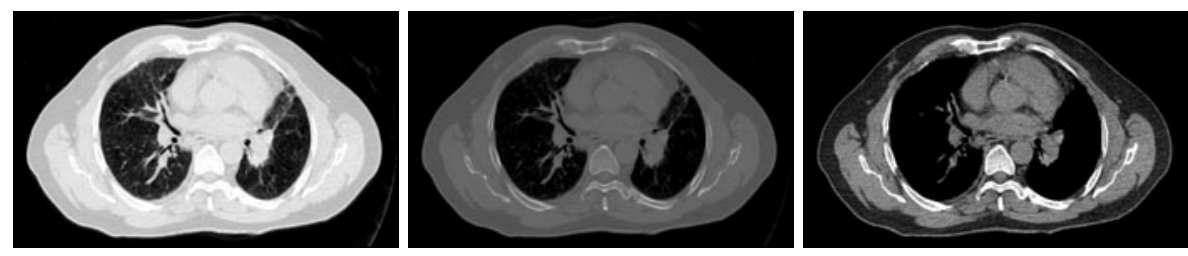

Fig. 1. An example from a CT chest scan displaying the use of different transfer functions. From left: Lung window, bone window and mediastinum window. 

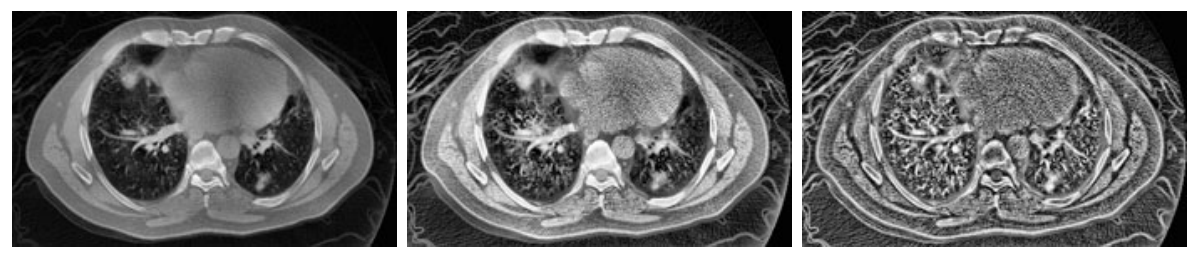

Fig. 2. An image from a CT chest scan displaying the use of CLAHE (adapthisteq in MATLAB) with various parameter settings

There are other approaches for handling this problem that do not include histogram equalization. In [7] it is suggested that the use of a bi-linear transfer function will improve the visualization. Another approach, presented in 4], is where multiple transfer functions are applied separately and then combined using weighted averaging. In a US patent application, 2, yet a third alternative is presented, where a single transfer function is applied first and later, two other transfer functions are applied to the values that were clipped due to the first transfer function. This approach resembles a functionality, known as dual window, that used to be available on some modality workstations. Dual window functionality allowed the radiologist to apply a contrast window within an already applied contrast window. However, a common limitation of these three suggestions is that they fail to make use of the fact that different transfer functions are often only relevant to disjoint regions.

In this paper, we present a method for the utilization of local transfer functions for visualization of medical image volumes based upon deformable models. The aim of this suggestion is to enable the radiologist to view images with multiple local transfer function applied simultaneously to the volume, instead of just one global transfer function. The transfer functions are automatically assigned to different anatomically relevant regions in the volume to be visualized and they can either be pre-defined or interactively changed by the radiologist during the visualization.

\section{Model-Based Local Transfer Function}

The basic idea behind the proposed method consists of an anatomical model that is deformed, with the aid of non-rigid registration, to fit the data volume to be visualized. Voxel-specific transfer functions are then applied to the volume, where the transfer functions are derived from the deformed model. This will enable the radiologist to review images while applying multiple transfer functions simultaneously. The applied model can be more or less complex depending on the number of segmented tissues (compartments) in the model, in which a predefined transfer function is linked to each compartment. The radiologist can also interactively control the transfer function for each compartment during the review process. See Fig. 3 for a graphical overview of the proposed method.

The following sections will in more detail describe the different aspects of the proposed method. 


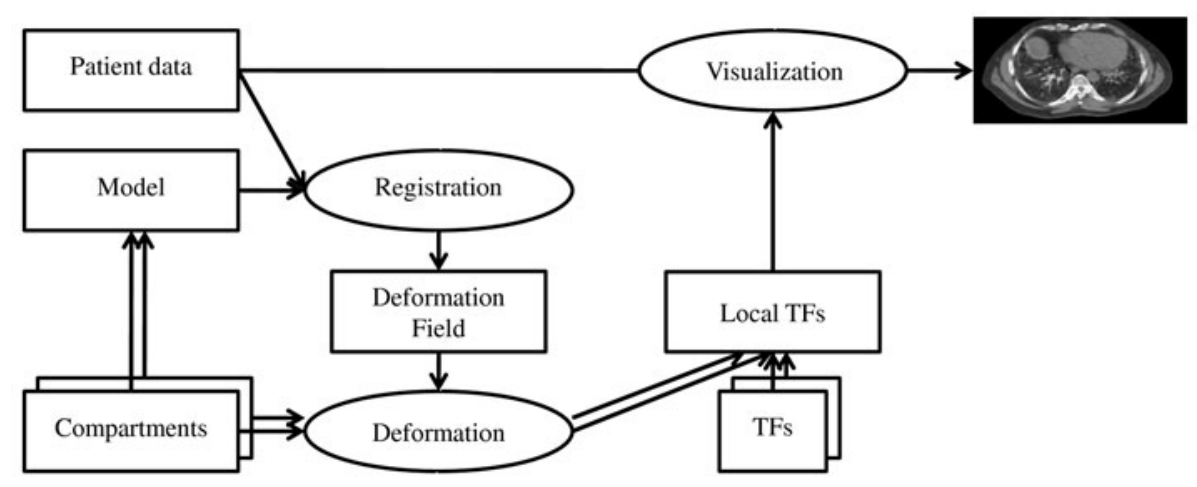

Fig. 3. Method overview

\subsection{Model}

The model $I_{M}$ consists of a body with $N$ compartments, where $I_{C_{i}}(\boldsymbol{x})$ describes the probability $[0,1]$ of tissue $i$ in $\boldsymbol{x}$. The different compartments are combined to create the model according to:

$$
I_{M}=\sum_{i=1}^{N} a_{i} I_{C_{i}}
$$

Where $\sum_{i=1}^{N} I_{C_{i}}(\boldsymbol{x})=1$ and where $a_{i}$ are values used to combine the different compartments $I_{C_{i}}$ in order to create a model suitable for image registration. How to select $a_{i}$ depends on which registration method that is used in the subsequent step, e.g. an intensity-based method would require that the voxel values of the model are similar to the voxel values of the volume to visualize whereas a phase-based method would require that the boundaries between the different compartments in the model have the same sign as in the volume to visualize. A min and max value $\left(\alpha_{i}\right.$ and $\left.\beta_{i}\right)$ are assigned to each compartment. These values are used to control the transfer functions, see Fig. 4.

\subsection{Registration}

A segmentation and registration algorithm known as the Morphon is employed to register the model with the data set to be visualized. The Morphon is a phasebased algorithm where a source image $I_{S}(\boldsymbol{x})$, in our case $I_{S}=I_{M}$, is iteratively deformed, $I_{D}(\boldsymbol{x})=I_{S}(\boldsymbol{x}+\boldsymbol{d}(\boldsymbol{x}))$, until it is sufficiently similar to a target image $I_{T}$. This process is performed over multiple scales starting on coarse scales to register large global displacements and moving on to finer scales to register smaller local deformations. For a more detailed review of the algorithm, the reader is referred to [8].

When the registration process is completed, the estimated deformation field is applied to all the compartments of the model using linear interpolation.

$$
\tilde{I}_{C_{i}}(\boldsymbol{x})=I_{C_{i}}(\boldsymbol{x}+\boldsymbol{d}(\boldsymbol{x}))
$$




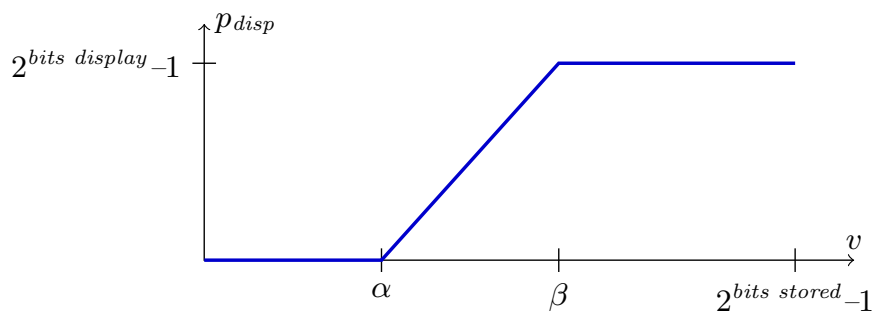

Fig. 4. An illustration of how the min and $\max$ values $\alpha$ and $\beta$ control the local transfer function, where $v$ is the local voxel value and $p_{\text {disp }}$ is the display value. Bits stored denotes the number of bits used to store each voxel value and where bits display denotes the number of bits available for display in the display device

\subsection{Visualization Based Upon Local Transfer Functions}

The next step is to define the voxel-specific transfer functions, which are defined by a min and max value for each voxel, $I_{\alpha}(\boldsymbol{x})$ and $I_{\beta}(\boldsymbol{x})$. The deformed compartments $\tilde{I}_{C_{i}}$ are combined for both the min and max values $\left(\alpha_{i}\right.$ and $\left.\beta_{i}\right)$ to create $I_{\alpha}(\boldsymbol{x})$ and $I_{\beta}(\boldsymbol{x})$.

$$
\begin{aligned}
I_{\alpha}(\boldsymbol{x}) & =\frac{\sum_{i=1}^{N} \alpha_{i} \tilde{I}_{C_{i}}(\boldsymbol{x})}{\sum_{i=1}^{N} \tilde{I}_{C_{i}}(\boldsymbol{x})} \\
I_{\beta}(\boldsymbol{x}) & =\frac{\sum_{i=1}^{N} \beta_{i} \tilde{I}_{C_{i}}(\boldsymbol{x})}{\sum_{i=1}^{N} \tilde{I}_{C_{i}}(\boldsymbol{x})}
\end{aligned}
$$

The data set to be visualized, $I$, is then transformed according to the voxelspecific transfer functions to form $I_{\text {disp }}$, which then is used for visualization.

$$
\begin{aligned}
I_{\text {slope }} & =\frac{2^{\text {bits display }}-1}{I_{\beta}-I_{\alpha}} \\
\tilde{I} & =\min \left(\max \left(I, I_{\alpha}\right), I_{\beta}\right) \\
I_{\text {disp }} & =I_{\text {slope }}\left(\tilde{I}-I_{\alpha}\right)
\end{aligned}
$$

\subsection{Smooth Transition Regions between Transfer Functions}

An important issue to consider and to handle correctly is that of the transition regions between the compartments, i.e. how to handle the transition between different transfer functions. This is important since no registration algorithm can produce perfect results and since the borders between different tissues are rarely binary. Thus, it is important to have a transition that does not introduce any new features that are disturbing to the radiologist reviewing the images.

A first step which handles the transition regions is already incorporated into the model, since the compartments are described with a continuous probability 
between $[0,1]$ instead of with a binary model. The effect of this on the transfer functions in the transitions regions can be seen in Fig. [5] and the effect on the final visualization result can be seen in Fig. 6.

Another approach is to provide the radiologist with a tool for controlling the transition regions. A simple but effective tool is to include averaging of the transition regions, in which the radiologist can select which averaging filter to apply and the size of the applied filter.

Other approaches for handling the transition regions were tested, including different non-linear mappings of the probability functions describing the compartments and different non-linear combinations of the transfer functions in the transition region. However, none of them added any significant improvements than the already described continuous model and the user-controlled averaging of the transition regions.

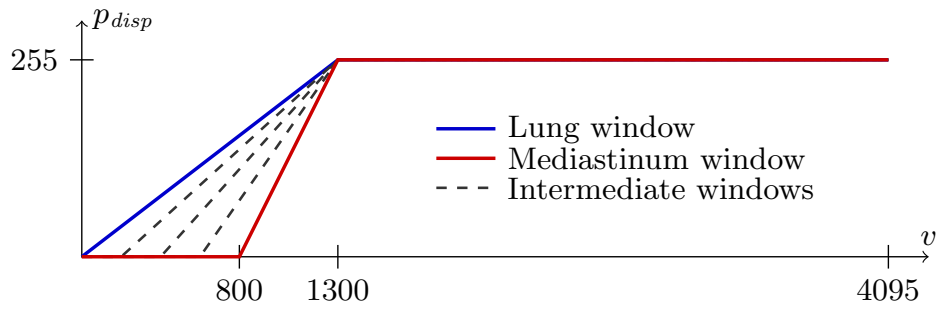

Fig. 5. An example to demonstrate how the transfer functions varies in the transition region between two different transfer functions
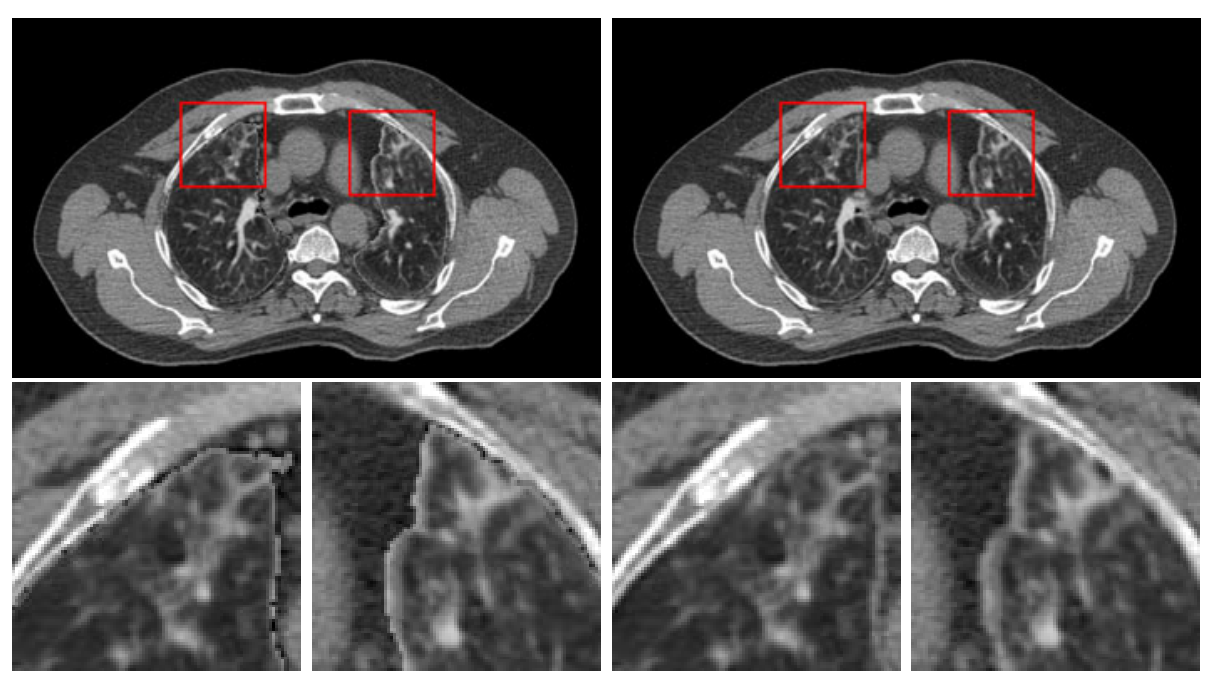

Fig. 6. One example to demonstrate the difference in using a binary model versus a continuous model. The close-ups especially highlights the difference in smoothness of the features introduced in the transition regions. Left side: Binary model, Right side: Continuous model. 


\section{Evaluation}

To evaluate the usefulness of the proposed method we have tested it on a number of clinical data sets and allowed three clinicians to evaluate the proposed method based upon the visualized images. The evaluation was based upon the following questions:

- What are the possibilities of the proposed method?

- What are the limitations of the proposed method?

- What is important to consider when handling transition regions, i.e. the region between two different transfer functions?

- Would it be possible to use the proposed method to make the review process of large medical image volumes more efficient?

\section{Implementation}

The proposed method was evaluated using data sets made available by [1 at http://www.dir-lab.com/. The data sets consist of 4D CT thoracic data from patients with esophageal cancer. Each 3D data set, in the 4D data sets, has a size of $512 \times 512 \times 128$ and a spatial resolution of $0.97 \times 0.97 \times 2.5 \mathrm{~mm}$. Using one of the patients, a model was created consisting of two compartments, see Fig. 7 . The model was then registered with two different data sets from the same patient but from different exhale/inhale phases. Each of the estimated deformation fields was applied to the model, which was then used to visualize the data sets using multiple transfer functions.
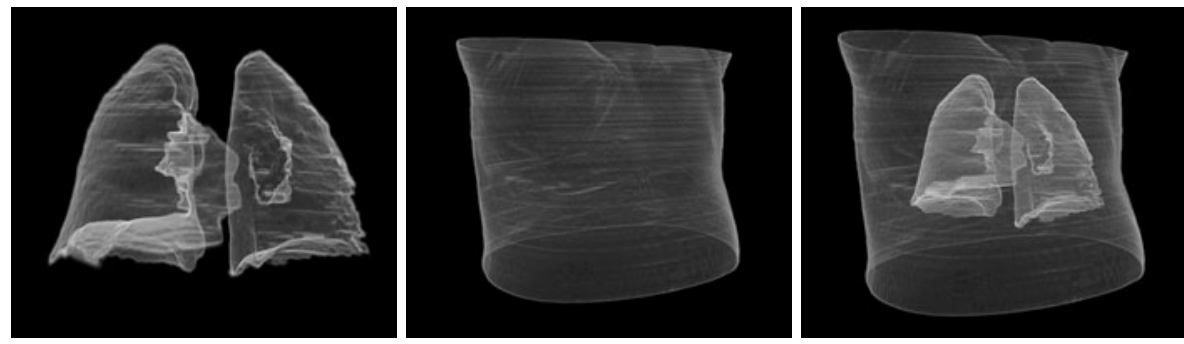

Fig. 7. Two different compartments (lung and body) combined to form the model

The model was created using MIPAV 5.0.0 and a semi-automated method to segment the contour of the body and of the lungs. A continuous model was achieved by applying Gaussian filtering to the binary segmentation of the body and of the lungs. In our case we set $a_{i}$ to 1 for the body compartment and to 0 for the lung compartment. The registration of the model with the data sets to be visualized was performed in MATLAB R2010a with the aid of a GPU-based implementation of the Morphon, implemented with CUDA 3.0. Due to memory 
constraints on the graphics card the data sets were downsampled to $256 \times 256 \times 128$ before registering the model with the data sets.

The deformation of the model and the visualization were performed in MeVisLab 4.6.2. Two different transfer functions were applied, one lung contrast window (min-max equal to $0-1300$ ) and one mediastinum contrast window (minmax equal to $800-1300)$. The display range for the visualized images was limited to eight bits, i.e. 256 gray-levels, since this is the commonly used display range when reviewing medical gray-level images.

\section{Results}

\subsection{Results from the Evaluation}

All three radiologists acknowledged the potential in the proposed method, in terms of making the review process more efficient by avoiding the need to
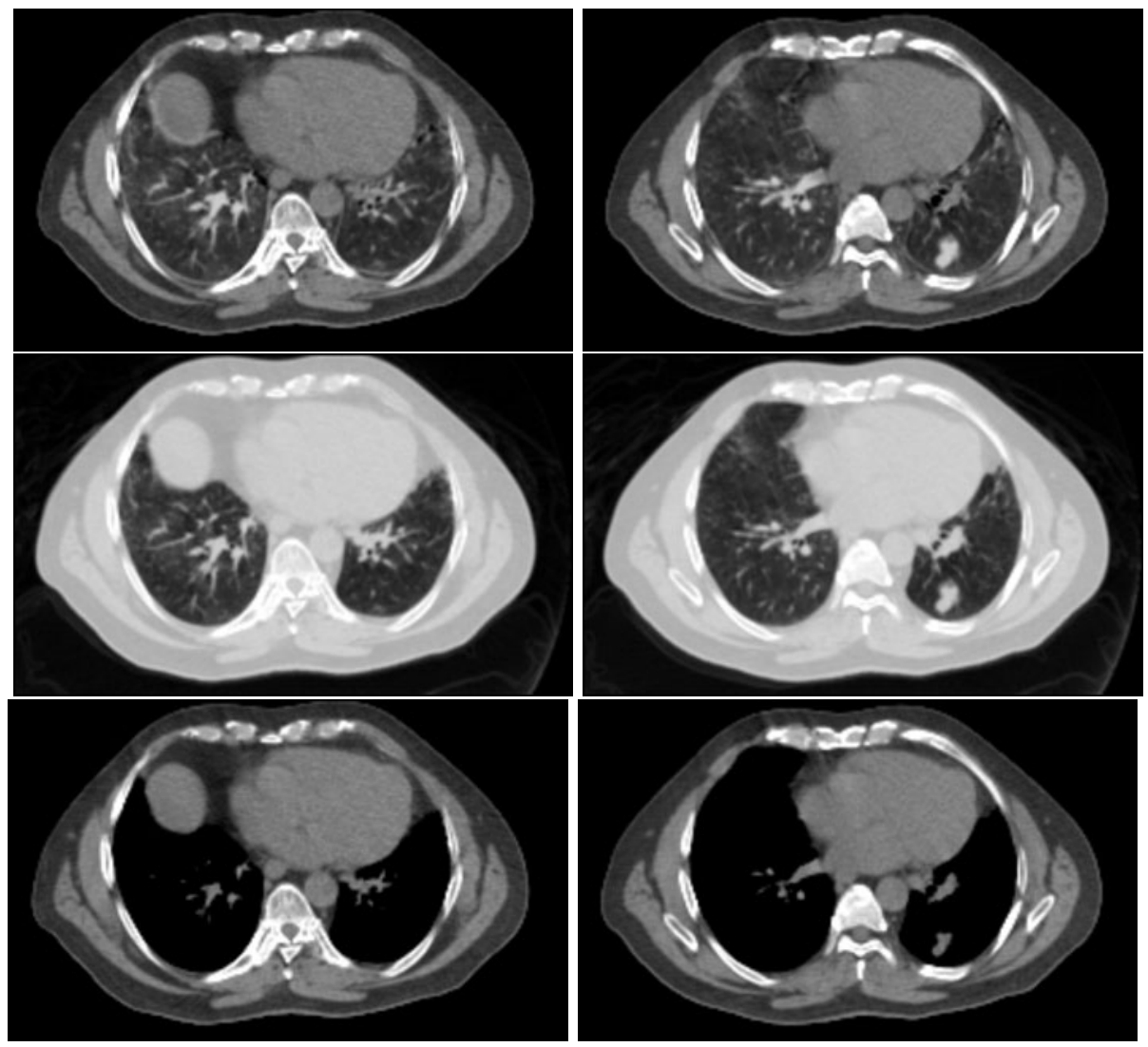

Fig. 8. Two examples to demonstrate the use of multiple transfer functions. Top row: Combined lung and mediastinum window, Middle row: Lung window, Bottom row: Mediastinum window. 
scroll through an image stack multiple times. They predicted that the method would have the greatest potential when working with images that display tissues/compartments where the visually important information is located within the tissues and not on the border between the tissues. Another type of relevant images would be those where there is a large difference between the applied transfer functions, e.g. transfer functions for air, soft tissues and/or bone. Another comment was that this method would be useful when there is a need for a quick overview, e.g. when the reason for the examination is vague or in trauma cases.

On the other hand, the radiologists found little or no potential in the proposed method, when reviewing images with small structures or where the borders between the different tissues/compartments contain vital information or when the applied transfer functions are very similar.
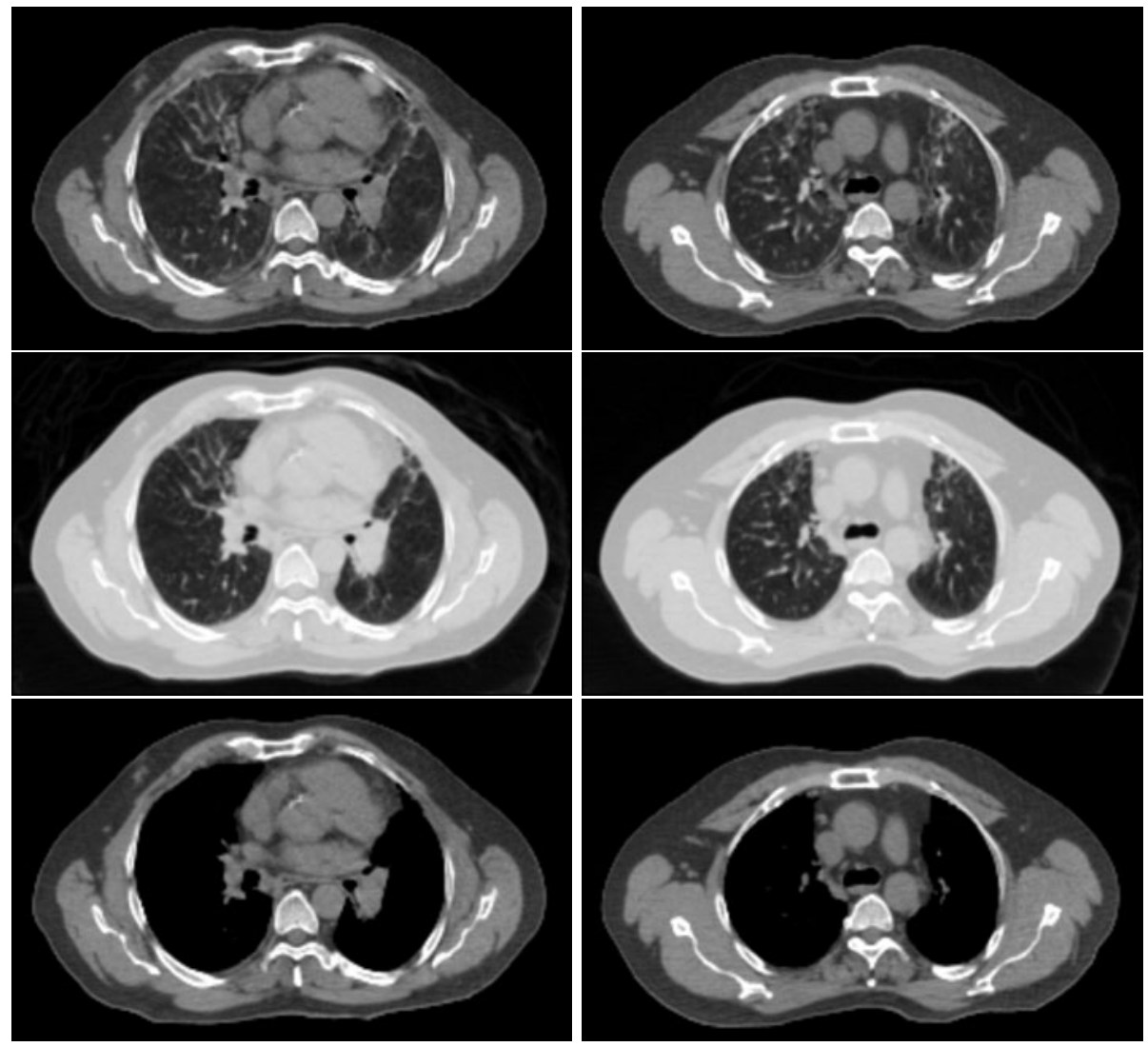

Fig. 9. Another two examples to demonstrate the use of multiple transfer functions.

Top row: Combined lung and mediastinum window, Middle row: Lung window, Bottom row: Mediastinum window. 
Another important comment from the radiologists, was that the images appeared familiar, albeit the simultaneous use of multiple transfer functions. Also the fact that the visualization was controlled using the familiar parameters of a contrast window, was highly appreciated.

The comments regarding how to handle the transition regions differed. One radiologist appreciated the smooth transition between the different transfer regions, because it did not visually disturb him as much as a sharp transition would, whereas the other two radiologists appreciated the sharp transition since it reminded them about the fact that multiple transfer functions where applied simultaneously.

\subsection{Results from the Implementation}

Results from the implementation of the proposed method can be seen in Figs. 8 and 9

\section{Discussion}

In this paper, we have presented a method for applying multiple transfer functions for visualizing gray-level images with a large dynamic range. This is possible since different transfer functions are often only relevant to disjoint anatomical regions, when radiologists review medical image volumes. To apply multiple transfer functions we have used a model, registered to the data set to be visualized, to control the local transfer function applied in each voxel.

The results presented, clearly indicate the usefulness of the proposed method, both in terms of its use of multiple transfer functions and its use of a registered model to control the applied transfer functions, in order to make the review process more efficient.

Two important features of the proposed method are that it does not introduce any new and unfamiliar parameters for the radiologists to work with and that, even though multiple transfer functions are applied simultaneously, voxels belonging to the same anatomical region are mapped with a single linear transfer function. The fact that these features are important and relevant to a radiologist was confirmed in the results from the evaluation in Sec. 5.1. This separates our method from the methods described in Sec. 1, where either both or one of these features are missing. For instance the histogram-based methods introduce an unfamiliar parameter set to work with and a non-linear mapping of the voxel values. The non-linear mapping of the voxel values is also introduced by the methods that attempts to visualize more by adapting the transfer functions.

However, there are a few aspects of the proposed method which can be commented upon. First, the method presented is dependent upon a successful registration of the model with the medical image volume to be visualized and especially that the deformation field, estimated by the registration process, is smooth. Thus, it is important to use registration methods that are diffeomorphic and that allows incorporation of prior knowledge to control the deformation field in order to improve the registration result, something which can be done using the 
Morphon, 35. Second, the reason for registering the model and not the actual patient data, upon which the model is based, is to avoid the risk of allowing details in the image volume to control the registration. Because of this, it was also natural to use the Morphon algorithm for the registration process, since, unlike other methods, it is phase-based and not intensity-based.

As previously described, we used a continuous probability of the different compartments in the model, in order to achieve a smooth transition region between different transfer functions, see Fig. 6. which would not introduce visually disturbing features to the radiologist whilst reviewing images. However, the results from the evaluation show, that there appears to exist a difference in opinion regarding how to handle the transitions regions. An individual accommodation to this, could easily be obtained by using the suggestion in Sec. 2.4. i.e. providing the user with functionality for smoothing the boundaries between the different compartments. Despite the use of a continuous model and various other attempts, new features were introduced in the transition regions, something which was difficult to completely avoid. However, this can be avoided if the voxel-specific transfer functions combined together form a global monotonically increasing transfer function. Since this would limit the contrast for each applied transfer function, it was not deemed relevant.

One of the key features of the proposed method is the use of local transfer functions, which has not been previously utilized in visualization of gray-level images. However, the notion of local or spatially dependent transfer functions is not entirely new. For instance, both [910] make use of spatially dependent transfer functions for direct volume rendering. A major difference, however, is that they use local histograms to control the local transfer functions instead of using a model which is registered to the volume to be visualized.

Future work includes creating more complex anatomical models and to evaluate examinations that include blood vessels with contrast, hip implants, trauma cases and brain scans. Furthermore, the proposed method needs to be evaluated in a user study involving a larger number of radiologists. Another future development would be to extend the method to incorporate transfer functions used in direct volume rendering.

\section{References}

1. Castillo, R., Castillo, E., Guerra, R., Johnson, V.E., McPhail, T., Garg, A.K., Guerrero, T.: A framework for evaluation of deformable image registration spatial accuracy using large landmark point sets. Physics in Medicine and Biology 54(7), 1849-1870 (2009)

2. Choi, R.J., F.L.R.: Multi-grayscale overlay window. US Patent Application Appl. No.: 12/175,308 (January 2010)

3. Forsberg, D., Andersson, M., Knutsson, H.: Adaptive anisotropic regularization of deformation fields for non-rigid registration using the morphon framework. In: ICASSP, Dallas, USA (March 2010)

4. Hidajat, N., Schroeder, R.J., Cordes, M., Felix, R.: Simultaneous presentation of soft tissue and bone tissue in computed tomography with combined window. Computers in Biology and Medicine 37(11), 1629-1636 (2007) 
5. Janssens, G., Jacques, L., de Xivry, J.O., Geets, X., Macq, B.: Diffeomorphic registration of images with variable contrast enhancement. International Journal of Biomedical Imaging (2010)

6. Jin, Y., Fayad, L.M., Laine, A.F.: Contrast enhancement by multiscale adaptive histogram equalization. Presented at the Society of Photo-Optical Instrumentation Engineers (SPIE) Conference.SPIE, vol. 4478, pp. 206-213 (December 2001)

7. John, A., Huda, W., Scalzetti, E.M., Ogden, K.M., Roskopf, M.L.: Performance of a single lookup table (LUT) for displaying chest CT images. Academic Radiology 11(6), 609-616 (2004)

8. Knutsson, H., Andersson, M.: Morphons: Paint on priors and elastic canvas for segmentation and registration, Joensuu (June 2005)

9. Lindholm, S., Ljung, P., Lundström, C., Persson, A., Ynnerman, A.: Spatial conditioning of transfer functions using local material distributions. IEEE Transactions on Visualization and Computer Graphics 16(6), 1301-1310 (2010)

10. Lundström, C., Ljung, P., Ynnerman, A.: Local histograms for design of transfer functions in direct volume rendering. IEEE Transactions on Visualization and Computer Graphics 12(6), 1570-1579 (2006)

11. Pizer, S.M.: Intensity mappings to linearize display devices. Computer Graphics and Image Processing 17(3), 262-268 (1981)

12. Pizer, S.M., Amburn, E.P., Austin, J.D., Cromartie, R., Geselowitz, A., Greer, T., Romeny, B.T.H., Zimmerman, J.B.: Adaptive histogram equalization and its variations. Comput. Vision Graph. Image Process 39, 355-368 (1987)

13. Zuiderveld, K.: Contrast limited adaptive histogram equalization, pp. 474-485. Academic Press Professional, London (1994) 